\title{
The Role of Bilirubin in Rheumatoid Arthritis as a Biomarker
}

\author{
Tuba Tülay Koca, 다이 Ejder Berk
}

Department of Physical Medicine and Rehabilitation, Sutcu Imam University Faculty of Medicine, Kahramanmaraş, Turkey

\section{ABSTRACT}

Objectives: Serum bilirubin $(\mathrm{Bb})$ has an anti-inflammatory biologic effect through its antioxidant component. The present study aims to reveal the importance of serum Bb in Rheumatoid arthritis (RA) as a biomarker.

Methods: This study was designed as a case-control study. Seventy-five RA patients with age $(p=0.31)$ and gender matched 75 controls were included in this study. Clinical characteristics and laboratory parameters were analyzed retrospectively. Serum direct $\mathrm{Bb}(\mathrm{DBb})$, indirect $\mathrm{Bb}(\mathrm{InBb})$, total $\mathrm{Bb}(\mathrm{TBb})$, erythrocyte sedimentation rate (ESR) and C-reactive protein (CRP) data were recorded. Disease activity in RA was assessed using the Disease Activity Score 28 (DAS28).

Results: In this study, acute phase reactants, CRP $(p<0.01)$ and ESR $(p<0.01)$ were significantly higher in our study group; $\mathrm{DBb}$ was found significantly lower $(p<0.01)$ than the control group. ESR was negatively correlated with $\mathrm{DBb}, \mathrm{InBb}$ and TBb respectively $(\mathrm{p}=0.029, r=-0.252 ; p=0.003 ; r=-0.362 ; p=0.009, r=-0.302)$. TBb was not correlated with DAS $28(\mathrm{p}=0.08 ; \mathrm{r}=-0.203)$.

Conclusion: We found that DBb was significantly lower in RA patients than the patients in the control group. All Bb parameters were negatively correlated with acute phase reactants and DAS28. As a practical biomarker with anti-oxidative properties, the DBb can be used in clinical follow-up in RA.

Please cite this article as: Koca TT, Berk E. The Role of Bilirubin in Rheumatoid Arthritis as a Biomarker. Anatol J Family Med 2019;2(3):98-102.

Address for correspondence: Dr. Tuba Tülay Koca. Department of Physical Medicine and Rehabilitation, Sutcu Imam University Faculty of Medicine, Kahramanmaraş, Turkey

Phone: +905063819295

E-mail:

tubatulaykoca@outlook.com

Received Date: 01.04.2019 Accepted Date: 08.04.2019 Published online: 13.11.2019 Journal of Family Medicine Available online at www.anatoljfm.org OPEN ACCESS

Keywords: Arthritis, rheumatoid, bilirubin, biomarkers

\section{INTRODUCTION}

Rheumatoid arthritis (RA) is a chronic autoimmune disorder that primarily affects the joints. RA typically results in hot, inflame, and painful joints. ${ }^{[1]}$ The course of disease in RA is irregular. An increasing number of studies show that besides the inherited genetic architecture, various environmental factors significantly contribute to the etiology of RA. ${ }^{[2]}$ In light of the diverse responses to targeted therapies, RA may represent a final common clinical phenotype that reflects many pathogenic pathways. ${ }^{[3]}$ Oxidative damage induced by reactive oxygen species has been related to the pathophysiology of RA in several studies, although results have been inconsistent and contradictory. ${ }^{[4]}$ Studies suggest that oxidative/ nitrosative stress markers may be useful in evaluating the progression of RA, as well as in elucidating the mechanisms of disease pathogenesis. ${ }^{[5]}$ Various biomarkers have been used for the prognosis and clinical follow-up. The identification of biomarkers that predict the treatment response before drug exposure is a current priority. Antibodies to citrullinated peptides, rheumatoid factor, and the interferon signature are the most robust and best-validated biomarkers identified to date. However, the range of biomarkers suitable for use in 
daily practice remains extremely narrow. ${ }^{[6]}$

Bilirubin $(\mathrm{Bb})$ is a heme degradation product with anti-inflammatory, antioxidative, and immunosuppressive features. ${ }^{[7]}$ Studies conducted in the previous two decades showed a strong antioxidative feature of $\mathrm{Bb}$ in addition to its being a degradation product. Many hypotheses were put forth concerning the mechanism of action of $\mathrm{Bb}$. The most well-known is the immunomodulatory effect that suppresses inflammation through its redox (antioxidative) capacity. ${ }^{[8]}$ The present study aims to reveal the clinical importance of serum $\mathrm{Bb}$ parameters in RA as a new biomarker in disease activity.

\section{METHOD}

This study was designed as a case-control study. Seventyfive RA patients with age and gender matched 75 controls were included randomly in this study. Clinical characteristics and laboratory parameters were analyzed. Some were new diagnoses; others were control subjects. In this study, 2010 American College of Rheumatology criteria was used for RA diagnosis. Disease activity of the patients with RA was assessed using the Disease Activity Score 28 (DAS28), C-reactive protein (CRP) and erythrocyte sedimentation rate (ESR). Disease Activity Score was calculated using the number of sensitive joints, the number of inflamed joints, visual analog scale and ESR.

Serum aspartate aminotransferase (AST), alanine aminotransferase $(A L T)$, total $B b(T B b)$, direct $B b(D B b)$, indirect $B b$ $(\mathrm{InBb})$ were among the biochemical parameters that were measured by automatic biochemical analysis.

The number of swollen and sensitive joints was identified through a physical examination. The Bb parameters were compared with all parameters. Patients with a history of liver dysfunction, chronic liver disease, liver damage and known liver function test abnormality, biliary disease (cholelithiasis, cholecystitis), acute and chronic infection, other inflammatory rheumatological diseases and malignancy were excluded from the study.

\section{Statistical Analysis}

Continuous variables were shown as mean \pm standard deviation, and categorical variables were presented as percentages. We used the Kolmogorov-Smirnov test to identify data normality. The correlation between two continuous variables was analyzed using the Spearman test. The independent samples t-test and Mann-Whitney $U$ tests were used to analyze data according to their distribution. Statistical analysis was performed using SPSS 16.0 (SPSS Inc.,
Chicago, IL, USA); $\mathrm{p}<0.05$ was designed as statistically significance. This study complied with Helsinki declaration principles and ethical rules.

\section{RESULTS}

Seventy-five (50.0\%) RA patients with age $(p=0.31)$ and gender $(p=0.06)$ matched $75(50.0 \%)$ controls were included in this study. The median number of tender joints was 6.0 (0.0-16.0); swollen joints were $0.0(0.0-6.0)$ in the study group. DAS28 of the study group was $3.8 \pm 1.0$. The descriptive and analytic data for the groups were given in Table 1 and Table 2, respectively. Acute phase reactants, CRP $(p<0.001)$ and ESR $(p<0.001)$ were significantly higher in our study group; DBb was found significantly lower than controls ( $p<0.001)$ (Fig. 1).

In correlation analysis, ESR was negatively correlated with $\mathrm{DBb}, \operatorname{InBb}$, and $\mathrm{TBb}$ respectively $(\mathrm{p}=0.029, \mathrm{r}=-0.252$;

\section{Table 1. Clinical parameters of the RA patients}

RA group $(\mathbf{n}=75)$

$\begin{array}{lc}\text { Number of tender joints } & 6.0(0.0-16.0) \\ \text { Number of swollen joints } & 0.0(0.0-6.0) \\ \text { Morning stiffness (minutes) } & 15.0(0.0-240.0) \\ \text { DAS28 } & 3.8 \pm 1.0 \\ \text { VAS (centimeter) } & 5.5 \pm 2.3\end{array}$

RA: rheumatoid arthritis; DAS 28: disease activity score; VAS: visual analog scale. Data were given mean \pm std or median (minimum-maximum) according to their distribution. All data are presented as mean \pm standard deviation or median (minimum-maximum), where appropriate.

Table 2. Analytic data of the groups

\begin{tabular}{lccc} 
& $\begin{array}{c}\text { RA group } \\
(\mathbf{n = 7 5 )}\end{array}$ & $\begin{array}{c}\text { Control group } \\
(\mathbf{n = 7 5 )}\end{array}$ & p \\
\hline Age (year) & $50.0 \pm 10.5$ & $51.9 \pm 9.3$ & 0.31 \\
Gender (M/F) & $32(42.7) / 43(57.3)$ & $40(53.3) / 35(46.7)$ & 0.06 \\
ESR (mm/h) & $29.5 \pm 18.3$ & $15.6 \pm 13.4$ & $<0.001$ \\
CRP (mg/dL) & $7.7 \pm 10.5$ & $1.1 \pm 1.6$ & $<0.001$ \\
TBb & $0.53 \pm 0.22$ & $0.52 \pm 0.22$ & 0.81 \\
DBb & $0.15 \pm 0.07$ & $0.19 \pm 0.01$ & $<0.001$ \\
InBb & $0.33 \pm 0.17$ & $0.37 \pm 0.17$ & 0.21 \\
AST & $21.4 \pm 2.6$ & $19.7 \pm 4.7$ & 0.19 \\
ALT & $24.2 \pm 7.02$ & $22.7 \pm 8.2$ & 0.07
\end{tabular}

ESR: erythrocyte sedimentation rate; CRP: C-reactive protein; TBb: total bilirubin; DBb: direct bilirubin; InBb: indirect bilirubin; AST: aspartate aminotransferase; ALT: alanine aminotransferase. 


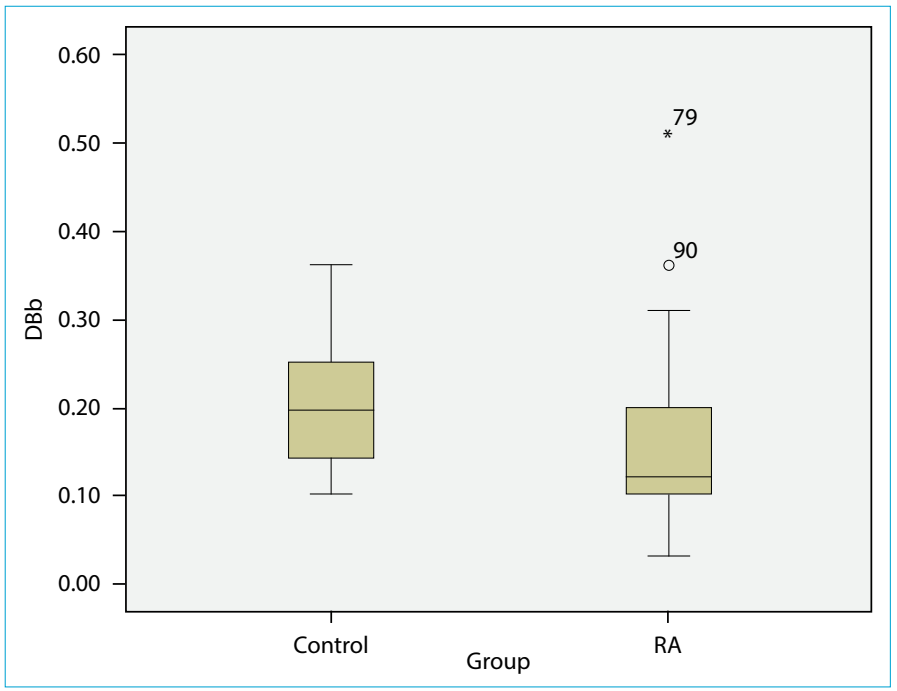

Figure 1. Boxplot of direct bilirubin according to the groups.

$p=0.003, r=-0.362 ; p=0.009, r=-0.302)$. TBb was also not correlated with DAS $28(p=0.08 ; r=-0.203)$.

\section{DISCUSSION}

RA is a chronic autoimmune disease that is characterized by widespread synovitis resulting in joint damage and erosion in the articular cartilage and marginal bone. ${ }^{[8]}$ RA pathophysiology shares the common cascades with atherosclerosis, including endothelial dysfunction related to the underlying chronic inflammation. ${ }^{[9]}$ Biomarkers indicating inflammation will assist during disease follow-up. ${ }^{[10]}$ Different biomarkers from several tissues (blood, urine, synovial fluid or tissue) and different structures (autoantibody, genetic marker, joint remodelling marker) have been studied for their diagnostic and prognostic importance in RA during the last few decades. ${ }^{[11]}$

The management of patients with autoimmune rheumatic diseases, such as $R A$, remains a significant challenge. We often concentrate on treating and relieving the symptoms and consequences and not the underlying causes of the disease. Oxidative stress may occur in many autoimmune diseases, with the excess production of reactive oxygen species (ROS) and reactive nitrogen species. ${ }^{[12]} \mathrm{It}$ is believed that ROS play a role in RA pathogenesis. Overproduction of ROS causes damage in lipids, proteins, and nucleic acids. ${ }^{[13,14]}$ Oxidative stress is positively correlated with disease severity for patients with RA. Reactive oxygen species is the activating signal transmission pathway for the inflammatory response in RA. Beginning from this point, we can say that an antioxidant defence system that is targeting ROS has an important role in RA pathogenesis. ${ }^{[15-17]}$

$\mathrm{Bb}$ is a major product of the intravascular component of the heme catabolic pathway. It was traditionally considered a metabolic product of heme that provided only a minimal contribution to human physiology and thought to be a degradation product. However, in the last two decades, antioxidative features contributing to defence against oxidative stress have been discovered. Only a few studies have shown the effects of $\mathrm{Bb}$ molecule on diseases, such as cardiovascular diseases, diabetes, cancer, autoimmune diseases (lupus, rheumatoid arthritis), and schizophrenia. ${ }^{[14,18,19]}$ We know that a physiologic dose of $\mathrm{Bb}$ is beneficial and also that toxic doses cause immunosuppression. Lee $Y$ et al. ${ }^{[17]}$ observed the inhibition of acute inflammation processes in the colon through experimental intravenous injection of $\mathrm{Bb}$ nanoparticles in rats with ulcerative colitis. Ample evidence has been found for a potent anti-inflammatory role for $\mathrm{Bb}$ as a degradation product of heme. ${ }^{[15-17]}$ Vogel ME et al. ${ }^{[18]}$ showed the effects of $\mathrm{Bb}$ in intestinal tissue via its disrupting eosinophil migration.

Contrarily, evidence of an increase in the severity of schizophrenia with increasing $\mathrm{Bb}$ values has been shown. However, several studies have also shown that $\mathrm{Bb}$ could be a protective factor for RA, multiple sclerosis, and systemic lupus erythematosus. ${ }^{[19]}$ In their NHANES study, Fischman et al. ${ }^{[20]}$ showed that $\mathrm{Bb}$ is a protective factor for RA. The development of RA has an inverse relationship with the serum TBb. This protective effect was linked to its physiologic anti-inflammatory effects resulting from the anti-oxidant feature of $\mathrm{Bb}^{\left[{ }^{[8]}\right.}$ Peng et al. ${ }^{[2]]}$ found that lower serum $\mathrm{Bb}$ values in all patients with polymyositis than healthy controls; a possible relationship with disease activity was also detected. Zheng J et al. ${ }^{[22]}$ found higher serum CRP values and lower TBb values in diabetic patients and patients with impaired insulin tolerance. For patients with psoriasis, a negative relation was found between TBb values and inflammatory parameters. ${ }^{[23]}$ Peng YF et al. ${ }^{[24]}$ detected lower $\mathrm{DBb}$ values and higher CRP values when assessing neurogenic inflammation in patients with migraine and CRP and $\mathrm{DBb}$ were also found negatively correlated. Beciragic $\mathrm{A}$ et al. ${ }^{[25]}$ did not find any relationship between higher CRP values and serum uric acid, ferritin, and $\mathrm{Bb}$. Koca et al. ${ }^{[26]}$ found low levels of $\mathrm{DBb}$ in patients with Ankylosing spondylitis and negatively correlated with disease activity similarly. We found a significant negative relation in $\mathrm{Bb}$ parameters with DAS 28 score and acute phase reactants. Also, DBb levels were found lower in RA patients than the patients in the control group; this may be explained by DBb decreases as the inflammation increases.

On the other hand, Bb parameters may be exhausted due to increased oxidative stress as the disease activity increases in RA patients. 
The decrease in $\mathrm{Bb}$ levels seems to be the result of the disease rather than an etiological factor.

There are some limitations to the study. The sample size is not enough to make certain generalizations. Our study group mainly consisted of patients in remission. Additionally, serum $\mathrm{Bb}$ concentration is affected by several factors, such as gender, smoking, medication, alcohol and anemia.

Liver enzymes, including $\mathrm{Bb}$ parameters, may be affected depending on the rheumatic diseases itself and the medications used. Not considering the medication of the participants is another limitation of this study.

To sum up, this study supports the anti-inflammatory feature of Bb similarly found in other studies in the literature. We found that DBb was significantly lower in RA patients than the patients in the control group. As a practical biomarker with anti-oxidative properties, the DBb can be used in clinical follow-up in RA.

\section{Disclosures}

Peer-review: Externally peer-reviewed.

Conflict of Interest: None declared.

Ethics Committee Approval: The study was approved by the Local Ethics Committee.

Authorship Contributions: Concept - T.T.K.; Design - E.B.; Supervision -T.T.K.; Materials -T.T.K.; Data collection \&/or processing - T.T.K.; Analysis and/or interpretation - E.B. ;T.T.K.; Literature search - T.T.K.; Writing -T.T.K.; Critical review - T.T.K.

\section{REFERENCES}

1. Majithia V, Geraci SA. Rheumatoid arthritis: diagnosis and management. Am J Med 2007;120(11):936-9. [CrossRef]

2. Glant TT, Mikecz K, Rauch TA. Epigenetics in the pathogenesis of rheumatoid arthritis. BMC Med 2014;12:35. [CrossRef]

3. Firestein GS. The disease formerly known as rheumatoid arthritis. Arthritis Res Ther 2014;16(3):114. [CrossRef]

4. García-González A, Gaxiola-Robles R, Zenteno-Savín T. Oxidative stress in patients with rheumatoid arthritis. Rev Invest Clin 2015;67(1):46-53.

5. Veselinovic M, Barudzic N, Vuletic M, Zivkovic V, Tomic-Lucic $A$, Djuric $D$, et al. Oxidative stress in rheumatoid arthritis patients: relationship to diseases activity. Mol Cell Biochem 2014;391(1-2):225-32. [CrossRef]

6. Lequerré $T$, Rottenberg P, Derambure C, Cosette P, Vittecoq O. Predictors of Treatment Response in Rheumatoid Arthritis. Joint Bone Spine 2018;86(2):151-8. [CrossRef]

7. Jangi S, Otterbein L, Robson $S$. The molecular basis for the immunomodulatory activities of unconjugated bilirubin. Int J Biochem Cell Biol 2013;45(12):2843-51. [CrossRef]

8. Khan NM, Poduval TB. Immunomodulatory and immunotox- ic effects of bilirubin: molecular mechanisms. J Leukoc Biol 2011;90(5):997-1015. [CrossRef]

9. Nishimura K, Sugiyama D, Kogata Y, Tsuji G, Nakawaza T, Kawano S, et al. Meta-analysis: diagnostic accuracy of anticyclic citrullinated peptide antibody and rheumatoid factor for rheumatoid arthritis. Ann Int Med 2007;146(11):797-808.

10. Chimenti MS, Triggianese P, Conigliaro P, Candi E, Melino G, Perricone R. The interplay between inflammation and metabolism in rheumatoid arthritis. See comment in PubMed Commons below. Cell Death Dis 2015;6:e1887. [CrossRef]

11. Sanmartí R, Gómez-Puerta JA. Biomarkers in rheumatoid arthritis. Rheumatol Clin 2011;6S3:S25-8.

12. Smallwood MJ, Nissim A, Knight AR, Whiteman M, Haigh R, Winyard PG. Oxidative stress in autoimmune rheumatic diseases. Free Radic Biol Med 2018;125:3-14. [CrossRef]

13. Mateen S, Moin S, Zafar A, Khan AQ. Redox signaling in rheumatoid arthritis and the preventive role of polyphenols. Clin Chim Acta 2016;463:4-10. [CrossRef]

14. Vítek L. Relationship of bilirubin to diseases caused by increased oxidative stress. Vnitr Lek 2013;59(7):618-21.

15. Cacciapaglia F, Anelli MG, Rizzo D, Morelli E, Scioscia C, Mazzotta $D$, et al. Influence of TNF-a inhibition on oxidative stress of rheumatoid arthritis patients. Reumatismo 2015;67(3):97102. [CrossRef]

16. Mateen S, Moin S, Khan AQ, Zafar A, Fatima N. Increased Reactive Oxygen Species Formation and Oxidative Stress in Rheumatoid Arthritis. PLoS One 2016;11(4):e0152925. [CrossRef]

17. Lee $Y$, Kim H, Kang S, Lee J, Park J, Jon S. Bilirubin Nanoparticles as a Nanomedicine for Anti-inflammation Therapy. Angew Chem Int Ed Engl 2016;55(26):7460-3. [CrossRef]

18. Vogel ME, Zucker SD. Biliburin acts as an endogenous regulator of inflammation by disrupting adhesion molecule-mediated leukocyte migration. Inflamm Cell Signal 2016;3(1). pii: e1178.

19. Isogai $H$, Hirayama N. A possible molecular mechanism of immunomodulatory activity of Bilirubib. Int J Med Chem 2013;2013:467383. [CrossRef]

20. Fischman D, Valluri A, Gorrepati VS, Murphy ME, Peters I, Cheriyath P. Bilirubin as a Protective Factor for Rheumatoid Arthritis: An NHANES Study of 2003 - 2006 Data. J Clin Med Res 2010;2(6):256-60. [CrossRef]

21. Peng YF, Zhang L, Pan GG, Wei YS. A potential clinical usefulness of measuring serum bilirubin levels in patients with polymyositis. Eur Rev Med Pharmacol Sci 2016;20(4):631-5.

22. Zheng J, Wu Y, Li Z, Wang H, Xiao W, Shi Y, Wang T. Low Serum Total Bilirubin Concentration was Associated with Increased High Sensitive C Reactive Protein Level in Patients with Impaired Glucose Tolerance and Type 2 Diabetes Mellitus Subjects. Clin Lab 2016;62(5):901-7. [CrossRef]

23. Zhou ZX, Chen JK, Hong YY, Zhou R, Zhou DM, Sun LY, et al. Relationship Between the Serum Total Bilirubin and Inflam- 
mation in Patients With Psoriasis Vulgaris. J Clin Lab Anal 2016;30(5):768-75. [CrossRef]

24. Peng YF, Xie LQ, Xiang Y, Xu GD. Serum Bilirubin and Their Association With C-Reactive Protein in Patients With Migraine. J Clin Lab Anal 2016;30(6):982-5. [CrossRef]

25. Beciragic A, Resic H, Prohic N, Karamehic J, Smajlovic A, Masnic
F, et al. Correlation Between C-reactive Protein and Non-enzymatic Antioxidants (Albumin, Ferritin, Uric Acid and Bilirubin) in Hemodialysis Patients. Mater Sociomed 2015;27(2):87-90.

26. Koca TT, Göğebakan H, Çetin GY. The clinic importance of bilirubin parameters in ankylosing spondylitis: Case control study. JOSAM 2018;2(3):330-3. [CrossRef] 